\title{
Heart Rate Characteristic Index Monitoring for Early Detection of Infections in Very Low Birth Weight Infants
}

\author{
Denise Moreira $^{1}$, Kristin Melton ${ }^{2,3}$, Jareen Meinzen-Derr ${ }^{4}$, Meredith Tabangin ${ }^{4}$, \\ Paul Kingma ${ }^{2,3}$, Kurt Schibler ${ }^{2,3, *}$ \\ ${ }^{1}$ Maternal-Infant, Department, Hospital Israelita Albert Einstein, Sao Paulo, Brazil \\ ${ }^{2}$ Perinatal Institute, Cincinnati Children's Hospital Medical Center, Cincinnati, USA \\ ${ }^{3}$ Department of Pediatrics, University of Cincinnati College of Medicine, Cincinnati, USA \\ ${ }^{4}$ Division of Biostatistics and Epidemiology, Cincinnati Children's Hospital Medical Center, Cincinnati, USA
}

\section{Email address:}

kurt.schibler@cchmc.org (K. Schibler)

${ }^{*}$ Corresponding author

\section{To cite this article:}

Denise Moreira, Kristin Melton, Jareen Meinzen-Derr, Meredith Tabangin, Paul Kingma, Kurt Schibler. Heart Rate Characteristic Index Monitoring for Early Detection of Infections in Very Low Birth Weight Infants. American Journal of Pediatrics.

Vol. 5, No. 3, 2019, pp. 170-174. doi: 10.11648/j.ajp.20190503.25

Received: July 16, 2019; Accepted: August 13, 2019; Published: August 28, 2019

\begin{abstract}
The aim of this study is 1) to determine the sensitivity and specificity of continuous heart rate characteristics (HRC) monitoring in detection of infections and 2) to evaluate whether HRC monitoring detects infections prior to onset of clinical symptoms in very low birth weight (VLBW) infants. A retrospective cohort study was conducted analyzing HRC scores and episodes of infection for VLBW infants in the Neonatal Intensive Care Unit (NICU) at Cincinnati Children's Hospital Medical Center from January 2015 through May 2016. HRC scores were acquired using the HRC monitor system and entered into the electronic medical record by bedside staff. Culture-positive and culture-negative clinical infections were recorded. Positive HRC scores were defined as an increase 1 point above the baseline or the first rise above 2 . HRC scores within 24 hours and also within the 5-day period before the start of antibiotics for infections were analyzed for sensitivity, specificity, positive predictive value and negative predictive value for detection of neonatal infection. The HRC score increase 1 point above the baseline or the first rise above 2 in the 24 hours before the start of antibiotics for infectious events had a sensitivity of $68.0 \%$, a specificity of $10.8 \%$. Positive predictive value (PPV) and negative predictive value (NPV) were $34.0 \%$ and 33.3\%, respectively. The PPV and NPV were modestly higher for elevated HRC scores during the 5-day period before infections, $41.1 \%$ and $66.7 \%$, respectively. In our single-center retrospective study, elevated HRC scores had limited ability to detect infection. More than half of the positive monitor events were not related to infection. The potential clinical impact of the monitor to detect infection before the onset of clinical symptoms was limited and the risk for unnecessary evaluation and treatment was high.
\end{abstract}

Keywords: HeRO Monitor, Neonatal Infection, Sepsis

\section{Introduction}

Improvement in neonatal care has enabled the survival of increasing numbers of very low birth weight (VLBW) infants [1]. As the number of VLBW infants grows, healthcare professionals face the struggle of not only decreasing mortality, but also improving quality of life. VLBW newborns are particularly susceptible to infections due their immature immune system [2]. Infections have a high rate of mortality and morbidity in this population [3]. Not only are infections associated with unfavorable outcomes in premature infants, their early diagnosis presents a challenge because symptoms are often subtle and nonspecific. Heart rate variability has been studied as a potential approach to enable detection of sepsis in infants prior to onset of clinical deterioration. Heart rate variability is a physiological finding regulated by the autonomic nervous system [4]. Its disturbance and correlation with different pathologies have 
been established [5-8]. A heart rate characteristics (HRC) score was developed based on diminished heart rate variability and transient decelerations observed in septic infants [9]. A device, called the $\mathrm{HeRO}^{\mathrm{tm}}$ monitoring system (Medical Predictive Science Corporation, Charlottesville VA), is commercially available that uses electrocardiograph findings to calculate the risk of developing a septic event within the next 24 hours. The use of this technology has been reported to reduce mortality in VLBW infants $[10,11]$. The objective of this study is to analyze if use of the Hero monitor improves early detection of sepsis in VLBW infants in a quaternary level Neonatal Intensive Care Unit. HRC monitoring is routinely used in all VLBW infants admitted to our NICU. The monitor provides non-invasive and continuous information on the probability of developing infection in the population of high risk for infection throughout NICU course. A rising score alerts the medical team of a possible onset of infection and prompts close clinical observation to determine if the patient should be evaluated for infection and started on antibiotic treatment. We hypothesized that these alerts would help to diagnose and treat infections earlier and before clinical deterioration.

\section{Methods}

A retrospective cohort study was designed to retrieve data from all VLBW infants $<1500$ grams who were submitted to HRC monitoring in the Neonatal Intensive Care Unit (NICU) at Cincinnati Children's Hospital Medical Center, a quaternary level facility. All patients were outborn. The study included patients admitted to the NICU during the period from January 2015 to May 2016. Approval from the Institutional Review Board was obtained. The suggested care of patients undergoing HRC monitoring in our unit is to clinically examine the patient when there is an increase of one point above his prior baseline or when the score rises above 2 for the first time. Based on the clinical evaluation, the provider decides if the patient should be tested for sepsis and started on antibiotics. Medical records were reviewed to identify HRC scores for each patient. The scores are generally recorded hourly in the electronic medical record for each patient. HRC monitoring was considered positive for each 24-hour period in which the score was either one point above baseline or it rose above 2 the first time. All available positive scores were recorded during data collection.

For infants who received evaluation for infection, data was collected on the presence of clinical symptoms as well as laboratory studies that were obtained for assessment, including CD64 measurement, bacterial cultures and radiographs. Consecutive days on antibiotics were recorded. Clinical symptoms that were assessed included hyper/ hypothermia, apnea, respiratory distress, need for increased respiratory support, feeding intolerance, abdominal distension, lethargy and hyperglycemia. CD64 index cut off for our laboratory is 2.3 [12]. Clinical infections in the absence of positive cultures were included if they prompted at least 5 days of antibiotics and were associated with evidence of a systemic inflammatory response [13]. They were categorized in two groups: culture proven infection when blood, urine, cerebrospinal fluid or peritoneal fluid were culture positive or clinical sepsis, such as necrotizing enterocolitis (NEC), pneumonia, ventilator associated pneumonia (VAP) and tracheitis. Data on elevated scores without further infection screening was also collected. It included searching for non-infectious causes for heart rate variability such as perinatal asphyxia, Grade 3 or 4 of intraventricular hemorrhage (IVH), surgery, retinopathy of prematurity exam or intervention.

To assess the diagnostic ability of the Hero monitor for early detection of sepsis in VLBW infants: sensitivity, specificity, positive predictive value and negative predicted value were calculated with $95 \%$ confidence intervals determined using the Wilson score method [14]. For infants with infection, classification of positive HRC scores were assessed at specified timepoints (24-hour and 5-day intervals) before the start of an antibiotic. For infants without infection, however, a predefined timepoint cannot be determined. Thus, for these infants, positive HRC scores were classified at any timepoint during data collection. Sensitivity and specificity calculations were subject to bias since the number of screening timepoints differ by group, in light of the continuous screening and calculations based on infection and not the individual. To mitigate bias, sensitivity and specificity were analyzed at the level of the infant using the first infection for those patients with multiple infections. SAS version 9.3 was used for all analyses.

\section{Results}

Characteristics of the study cohort are presented in Table 1.

Table 1. Study cohort characteristics $(N=62)$.

\begin{tabular}{ll}
\hline Characteristic & Values \\
\hline Female Sex, N (\%) & $27(43.6)$ \\
Birth Weight Median, (Range) & $780(479-1460)$ \\
Gestational Age Median, (Range) & $27(23-34)$ \\
Age at NICU Admission (days), Median (Range) & $7(0-96)$ \\
NICU days, Median (Range) & $57(2-202)$ \\
HERO monitoring days, Median (Range) & $57(1-123)$ \\
Deaths, N (\%) & $12(19.4)$ \\
Age at death (days), Median (Range) & $75(2-230)$ \\
\hline
\end{tabular}

Indications for transfer to this quaternary care NICU include preterm delivery at a regional delivery hospital requiring neonatology services or the need for pediatric subspecialty care such as cardiology, genetics, neurosurgery or pediatric surgery. All $62 \mathrm{VLBW}$ infants received HRC monitoring as a component of their care during the study period.

A total of 73,808 scores were registered in the electronic medical record over a median of 54 days (range 1 to 123 days). Of these, 2730 scores were linked to patients with missing data and were therefore excluded from analysis. Only $15 \%$ of HRC monitored days had at least one positive score (508 in 3356). Infection was the most frequent event 
related to HRC score increase as $45 \%$ of the positive days occurred during treatment for culture positive or clinical infection. Non-infectious events associated with positive HRC scores included 5 minutes Apgar score under 7 (39\%), same day medical procedure $(15 \%)$, and grade 3 or 4 IVH $(10 \%)$.

Fifty-one infectious events occurred in 25 patients which included 19 culture-proven infections and 32 episodes of clinical infection. Of culture-positive infections 9 cases were bloodstream infection, 9 cases were urinary tract infections, and there was one case of peritonitis caused by Candida albicans isolated from peritoneal fluid obtained during laparotomy. Pathogens detected in culture-proven infections are listed in Table 2.

Table 2. Pathogen representation among infants with culture-proven infections.

\begin{tabular}{llll}
\hline \multirow{2}{*}{ Agent } & \multicolumn{3}{l}{ Specimen } \\
\cline { 2 - 4 } & Blood & Urine & Peritoneal Fluid \\
\hline Candida albicans & 1 & 1 & 1 \\
Candida lusitaniae & 1 & & \\
Candida tropicalis & & 1 & \\
Enterobacter species & & 2 & \\
\hline
\end{tabular}

\begin{tabular}{llll}
\hline \multirow{2}{*}{ Agent } & \multicolumn{2}{l}{ Specimen } & \\
\cline { 2 - 4 } & Blood & Urine & Peritoneal Fluid \\
\hline Enterococcus faecalis & 1 & 1 & \\
Escherichia coli & & 1 & \\
Klebsiella pneumoniae & 2 & 1 & \\
Pseudomonas aeruginosa & & 1 & \\
Serratia marcescens & & 1 & \\
Staphylococcus aureus & 1 & & \\
Staphylococcus epidermidis & 1 & & \\
Streptococcus agalactiae & 1 & & \\
Streptococcus species & 1 & & \\
\hline
\end{tabular}

No cases of meningitis were detected. Cases of clinical infection included clinical sepsis (5), NEC (10), peritonitis (1), pneumonia (2), tracheitis (13) and ventilator-associated pneumonia (1).

We compared infants at their first episode of infection $(n=25)$ to those with no episodes of infection $(n=37)$. Among 25 infants with infections there were 11,293 HRC scores and of these 503 HRC scores were positive within the 24-hour interval and 2,442 HRC scores were positive within the 5-day interval preceding infection and antibiotic administration, Figure 1.

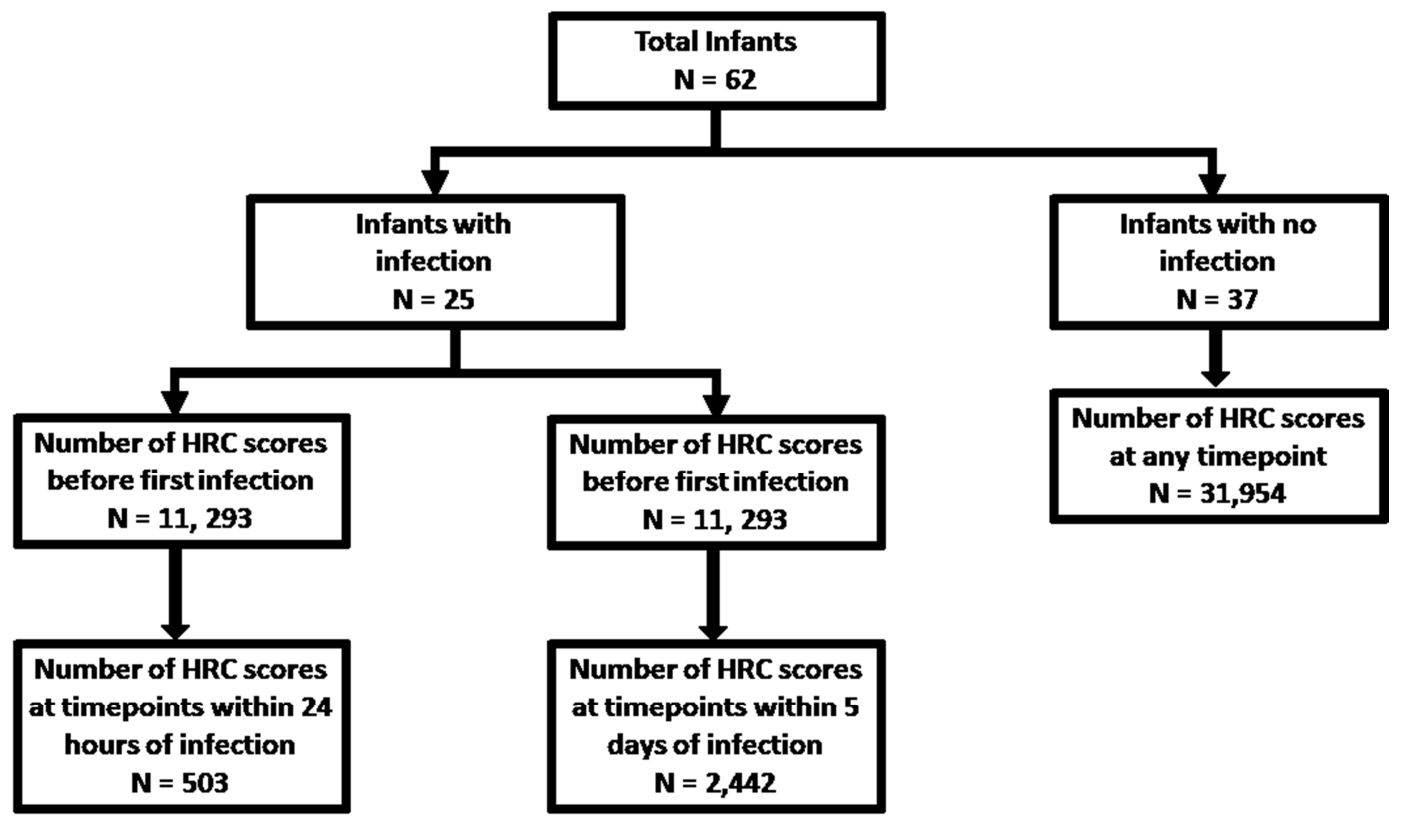

Figure 1. Flow diagram of HRC scores and diagnoses (n).

There were 31,954 HRC scores recorded at any time point among the 37 infants who did not develop infection. When the HRC scores were positive within 24 hours of the first episode of infection timed by antibiotic administration the monitor performed with a sensitivity of $68 \%(95 \%$ confidence Intervals, CI; $48.4 \%, 82.8 \%$ ) a specificity of $10.8 \%(\mathrm{CI} ; 4.3 \%, 24.7 \%)$, a positive predictive value of $34.0 \%(\mathrm{CI} ; 22.4 \%, 47.8 \%)$, and a negative predictive value of $33.3 \%$ [CI; $13.8 \%, 60.9 \%$ ] (Table 3 ).

Table 3. Performance of HRC scores in detecting the first infection episode within 24 hours of antibiotic administration and within five days of antibiotic administration.

\begin{tabular}{llll}
\hline $\begin{array}{l}\text { HRC one point above baseline OR first rise } \\
\text { above } \mathbf{2} \text { in } \mathbf{2 4} \text { hours before antibiotic start }\end{array}$ & Infection & No infection & $\begin{array}{l}\text { Estimates with 95\% Confidence Intervals (Wilson } \\
\text { Method) }\end{array}$ \\
\hline Screen positive, $\mathrm{N}$ & 17 & $33^{*}$ & Sensitivity: 68.0 (48.4, 82.8) Specificity: 10.8 (4.3, 24.7) \\
Screen negative, N & 8 & 4 & Positive Predictive Value: $34.0(22.4,47.8)$ Negative \\
Total Infants & 25 & 37 & Predictive Value: $33.3(13.8,60.9)$ \\
\hline
\end{tabular}




\begin{tabular}{llll}
\hline $\begin{array}{l}\text { HRC one point above baseline OR first rise } \\
\text { above } \mathbf{2} \text { in } \mathbf{5} \text { days before antibiotic start }\end{array}$ & Infection & No infection & $\begin{array}{l}\text { Estimates with 95\% Confidence Intervals (Wilson } \\
\text { Method) }\end{array}$ \\
\hline Screen positive & 23 & $33^{*}$ & Sensitivity: 92.0 (75.0, 97.8 Specificity: 10.8 (4.3, 24.7) \\
Screen negative & 2 & 4 & Positive Predictive Value: 41.1 (29.2, 54.1) Negative \\
Total Infants & 25 & 37 & Predictive Value: $66.7(30.0,90.3)$ \\
\hline
\end{tabular}

* Note: Screen positive at any timepoint.

When the HRC scores were positive within a 5-day interval of antibiotic administration HRC monitoring performed with a sensitivity of $92.0 \%(\mathrm{CI} ; 75.0 \%, 97.8 \%)$, a specificity of $10.8 \%(\mathrm{CI} ; 4.3 \%, 24.7 \%)$, a positive predictive value of $41.1 \%(\mathrm{CI} ; 29.2 \%, 54.1 \%)$, and a negative predictive value of $66.7 \%(\mathrm{CI} ; 30.0 \%, 90.3 \%)$.

\section{Discussion}

This study analyzed the performance of the HRC monitoring index in a quaternary level NICU. The index was developed comparing patients with sepsis and sepsis-like illness (negative blood culture) versus the patients without sepsis $[9-11,15]$. In the hands of these investigators the use of bedside HRC monitoring as a predictive tool of sepsis in preterm neonates was associated with decrease risk of mortality. Presumably, recognition of autonomic dysfunction, measured by HRC, may have led to earlier diagnosis of sepsis prompting earlier initiation of therapies, shortening the duration of organ dysfunction and reducing mortality. While our study was not powered to examine mortality, the HRC monitor performed poorly as a screening test in our population using a threshold level one point above baseline or first rise above 2, at both 24-hour and 5-day intervals before onset of infection. The low positive and negative predictive values at both time intervals reflect the continuous screening for a relatively rare event.

Predictive monitoring of $\mathrm{HRC}$ is a potential tool to improve outcomes in infected VLBW patients. It can be obtained continuously and non-invasively through electrocardiogram recordings that are already routinely monitored in the NICU setting. Reportedly, variation in HRC is prevalent in pediatric sepsis and correlates with disease severity and trajectory [16]. However, HRC monitoring had poor predictive capabilities for neonates in our quaternary unit. This might be due in part to the high prevalence of morbidities and procedures in our unit that elevate HRC scores thus limiting their utility. In addition, HRC is known to vary with factors such as age, gender, medications, and mechanical ventilation [17-20]. Nevertheless, a tighter protocol regarding the action towards patients with increasing scores might clarify the utility of HRC monitoring in early identification of infections in our NICU patients. It is likely that a protocol mandating clinical response to elevated HRC scores would increase laboratory testing and antibiotic usage as a secondary effect.

There were several limitations to our study inherent in its design using a retrospective review of the medical records. First, precise timing of events and discussions how HRC scores were incorporated into clinical decision-making were not discernable from review of the medical records. For example, this study was not able to measure the impact of increase in awareness towards a patient with a rising score. Sepsis symptoms in newborn infants are subtle and nonspecific during early phases. The presence of only one symptom will rarely trigger a screening for infection but a combination often will. The HeRO monitor is one more parameter that can help raise awareness for the possibility of infection. Second, there is not a mandatory intervention for a rising score. A positive score might have increased awareness, but not necessarily prompted immediate infection screening or initiation of antibiotic therapy. Third, the study population included a relatively small number of outborn VLBW infants referred to a regional quaternary center. The cohort included infants with complications of prematurity including spontaneous intestinal perforation, necrotizing enterocolitis, post-hemorrhagic hydrocephalus and chronic lung disease requiring subspecialty care. The incidence of bloodstream infection in the VLBW cohort in this study was $15 \%$, lower than what was previously reported $(21 \%)$ by the NICHD Neonatal Research Network; however, other sites of infection were identified in our study cohort. The mortality rate was higher than that reported in the literature, $19.4 \%$ compared to $10 \%^{3}$. This finding reflects the risk of a referral population, referred for surgical issues, and the known risks for community-born VLBW infants [21-24]. The utility of HRC scoring reported in our study might not be broadly generalizable to care for VLBW infants in other hospital settings since our center is a referral hospital and not a delivery hospital.

Studies to improve on the predictive capabilities of HRC monitoring are ongoing. Investigators are currently evaluating models combining HR and oxygen saturation variables in early detection of infection [25]. These investigators report a combined model (mean $\mathrm{SpO} 2$, standard deviation of $\mathrm{HR}$, and cross-correlation of HR-SpO2) provided additive value to an established HR characteristics index for illness prediction. Other measures of autonomic nervous system function such as respiratory rate variability, continuous temperature monitoring, automated pupillometry, and non-invasive baroreflex and chemoreflex sensitivity could potentially have application as effective biomarkers in neonatal sepsis, but further study is necessary [26]. Translating these metrics to real-time bedside displays and testing their impact on outcomes in randomized clinical trials is an essential next step.

\section{Conclusion}

In our single-center retrospective study conducted, 
elevated HRC scores had poor predictive capabilities to detect infection. More than half of the positive monitor events were not related to infection. Although the utility of HRC scoring reported in our study might not be broadly generalizable to care for VLBW infants in other hospital settings, the potential clinical impact of the monitor to detect infection before the onset of clinical symptoms in our quaternary level NICU was limited and the risk for unnecessary evaluation and treatment was high.

\section{References}

[1] Patel RM, Kandefer S, Walsh MC, et al. Causes and timing of death in extremely premature infants from 2000 through 2011. N Engl J Med. 2015; 372 (4): 331-340.

[2] Clapp DW. Developmental regulation of the immune system. Semin Perinatol. 2006; 30 (2): 69-72.

[3] Stoll BJ, Hansen N, Fanaroff AA, et al. Late-onset sepsis in very low birth weight neonates: the experience of the NICHD Neonatal Research Network. Pediatrics. 2002; 110 (2 Pt 1): 285-291.

[4] Heart rate variability. Standards of measurement, physiological interpretation, and clinical use. Task Force of the European Society of Cardiology and the North American Society of Pacing and Electrophysiology. Eur Heart J. 1996; 17 (3): 354-381.

[5] Vergales BD, Zanelli SA, Matsumoto JA, et al. Depressed heart rate variability is associated with abnormal EEG, MRI, and death in neonates with hypoxic ischemic encephalopathy. Am J Perinatol. 2014; 31 (10): 855-862.

[6] Verklan MT, Padhye NS. Heart rate variability as an indicator of outcome in congenital diaphragmatic hernia with and without ECMO support. J Perinatol. 2004; 24 (4): 247-251.

[7] Kovatchev BP, Farhy LS, Cao H, Griffin MP, Lake DE, Moorman JR. Sample asymmetry analysis of heart rate characteristics with application to neonatal sepsis and systemic inflammatory response syndrome. Pediatr Res. 2003; 54 (6): 892-898.

[8] Sullivan BA, Grice SM, Lake DE, Moorman JR, Fairchild $\mathrm{KD}$. Infection and other clinical correlates of abnormal heart rate characteristics in preterm infants. J Pediatr. 2014; 164 (4): 775-780.

[9] Griffin MP, O'Shea TM, Bissonette EA, Harrell FE, Jr., Lake DE, Moorman JR. Abnormal heart rate characteristics preceding neonatal sepsis and sepsis-like illness. Pediatr Res. 2003; 53 (6): 920-926.

[10] Fairchild KD, Schelonka RL, Kaufman DA, et al. Septicemia mortality reduction in neonates in a heart rate characteristics monitoring trial. Pediatr Res. 2013; 74 (5): 570-575.

[11] Moorman JR, Carlo WA, Kattwinkel J, et al. Mortality reduction by heart rate characteristic monitoring in very low birth weight neonates: a randomized trial. J Pediatr. 2011; 159 (6): 900-906 e901.
[12] Lynema S, Marmer D, Hall ES, Meinzen-Derr J, Kingma PS. Neutrophil CD64 as a diagnostic marker of sepsis: impact on neonatal care. Am J Perinatol. 2015; 32 (4): 331-336.

[13] Garner JS, Jarvis WR, Emori TG, Horan TC, Hughes JM. CDC definitions for nosocomial infections, 1988. Am J Infect Control. 1988; 16 (3): 128-140.

[14] Newcombe RG. Two-sided confidence intervals for the single proportion: comparison of seven methods. Stat Med. 1998; 17 (8): 857-872.

[15] Griffin MP, Moorman JR. Toward the early diagnosis of neonatal sepsis and sepsis-like illness using novel heart rate analysis. Pediatrics. 2001; 107 (1): 97-104.

[16] Ellenby MS, McNames J, Lai S, et al. Uncoupling and recoupling of autonomic regulation of the heart beat in pediatric septic shock. Shock. 2001; 16 (4): 274-277.

[17] De Rogalski Landrot I, Roche F, Pichot V, et al. Autonomic nervous system activity in premature and full-term infants from theoretical term to 7 years. Auton Neurosci. 2007; 136 (1-2): 105-109.

[18] El-Lakany MA, Fouda MA, El-Gowelli HM, El-Gowilly SM, El-Mas MM. Gonadal hormone receptors underlie the resistance of female rats to inflammatory and cardiovascular complications of endotoxemia. Eur J Pharmacol. 2018; 823: 41-48.

[19] Chang YT, Huang WC, Cheng CC, et al. Effects of epinephrine on heart rate variability and cytokines in a rat sepsis model. Bosn J Basic Med Sci. 2018.

[20] Thungtong A, Knoch MF, Jacono FJ, Dick TE, Loparo KA. Periodicity: A Characteristic of Heart Rate Variability Modified by the Type of Mechanical Ventilation After Acute Lung Injury. Front Physiol. 2018; 9: 772.

[21] Binder S, Hill K, Meinzen-Derr J, Greenberg JM, Narendran V. Increasing VLBW deliveries at subspecialty perinatal centers via perinatal outreach. Pediatrics. 2011; 127 (3): 487493.

[22] Wyckoff MH, Perlman JM. Effective ventilation and temperature control are vital to outborn resuscitation. Prehosp Emerg Care. 2004; 8 (2): 191-195.

[23] Koksal N, Baytan B, Bayram Y, Nacarkucuk E. Risk factors for intraventricular haemorrhage in very low birth weight infants. Indian J Pediatr. 2002; 69 (7): 561-564.

[24] Okuyama H, Ohfuji S, Hayakawa M, et al. Risk factors for surgical intestinal disorders in VLBW infants: Case-control study. Pediatr Int. 2016; 58 (1): 34-39.

[25] Fairchild KD, Lake DE, Kattwinkel J, et al. Vital signs and their cross-correlation in sepsis and NEC: a study of 1,065 very-low-birth-weight infants in two NICUs. Pediatr Res. 2017; 81 (2): 315-321.

[26] Badke CM, Marsillio LE, Weese-Mayer DE, Sanchez-Pinto LN. Autonomic Nervous System Dysfunction in Pediatric Sepsis. Front Pediatr. 2018; 6: 280. 